\title{
Indicadores antropométricos en dos escuelas públicas de zona rural y citadina en el Perú. 2014
}

\author{
Pedro Javier Navarrete Mejía ${ }^{1}$, Juan Carlos Velasco Guerrero ${ }^{1}$, Manuel Jesús Loayza Alarico ${ }^{1}$, Zoel Anibal \\ Huatuco Collantes ${ }^{2}$
}

\section{RESUMEN}

La desnutrición y la obesidad/sobrepeso asociados a una malnutrición generan la vulneración de las capacidades potenciales en el desarrollo físico y educativo en la etapa escolar. La Encuesta Nacional de Hogares del año 2008 evidencia información suficiente para asegurar que la desnutrición es de mayor impacto en zonas rurales y que la obesidad/sobrepeso es de mayor en las zonas urbanas. Durante estos últimos años el Estado Peruano en el marco de su modernización y crecimiento económico ha venido asignando importantes recursos a programas de asistencia alimentaria y nutricional a niños en etapa escolar.

Objetivo: Comparar indicadores antropométricos en dos escuelas primarias de una zona rural y citadina de características similares para determinar la tendencia de desnutrición y sobrepeso en estas zonas.

Material y Métodos: Estudio analítico, prospectivo de corte transversal - comparativo. La investigación incluyó 159 escolares del distrito de Acobamba (Huancavelica) y 280 escolares del distrito de Carabayllo (Lima) procedentes de dos escuelas públicas de una zona citadina y rural. Se recolectó información sobre las variables de estudio (edad, sexo, peso, talla e IMC) mediante una ficha pre elaborado. El periodo de recolección de la información fue entre abril y mayo de 2014.

Resultados: Se evidenció mayor prevalencia de desnutrición en Acobamba (10.06\%) y mayor Obesidad/Sobrepeso en Carabayllo (16.79\%); las medias de IMC de los niños(as) de Acobamba y Carabayllo son estadísticamente iguales.

Conclusión: Las características epidemiológicas de la población estudiada tanto en los distritos de Acobamba (Huancavelica) como Carabayllo (Lima) muestra relación de la desnutrición en zonas rurales, mientras que la obesidad/ sobrepeso a pesar de ser dos poblaciones con características geográficas diferentes (urbana y rural) presentan características similares en torno al estado nutricional de su población, para la muestra de estudio, lo cual indicaría cambios en los perfiles de consumo de alimentación en las zonas rurales, por lo que es necesario realizar estudios para conocer los factores de este incremento. (Horiz Med 2015; 15(4): 6-10)

Palabras clave: desnutrición, estado nutricional, escolares. (Fuente: DeCS BIREME).

\section{Anthropometric indicators in two public schools of urban and rural zones in Peru. 2014}

\begin{abstract}
Malnutrition and obesity / overweight associated with malnutrition generate infringement of the potential capabilities on the physical and educational development at school age. The National Household Survey 2008 is sufficient evidence to ensure that malnutrition has the greatest impact on rural areas and that obesity / overweight is higher in urban areas. In recent years the Peruvian government, as part of its modernization and economic growth, has been allocating significant resources to programs for food and nutritional assistance to school aged children in.

Objetive: Compare anthropometric indicators in two primary schools in a rural and urban area with similar characteristics, to determine the trend of malnutrition and overweight in these areas.

Material and Methods: Analytical- comparative, prospective cross-sectional study. The research included 159 children form the Acobamba school district (Huancavelica) and 280 children from the Carabayllo school district (Lima) from two public schools in an inner city and rural area. Information on the study variables (age, sex, weight, height and BMI) were collected using a pre prepared data collection sheet. The period of data collection was from April to May 2014

Results: higher prevalence of malnutrition was evident in Acobamba (10.06\%) and increased obesity/overweight in Carabayllo (16.79\%); the mean BMI of children of Acobamba and Carabayllo are statistically equal.

Conclusion: The epidemiological characteristics of the study population in both districts of Acobamba (Huancavelica) and Carabayllo (Lima) show relationship of malnutrition in rural areas, while obesity / overweight. Despite being two populations with different geographical characteristics (urban and rural) they have similar characteristics around the nutritional status of the population, for the study sample, which would indicate changes in the profiles of food consumption in rural areas, so it is necessary to conduct studies to determine the factors of this increase. (Horiz Med 2015; 15(4): 6-10)
\end{abstract}

Key words: malnutrition, nutritional status, school age children. (Source: MeSH NLM).

Doctor en Salud Pública, Facultad de Medicina-Universidad de San Martin de Porres

Doctor en Medicina, Facultad de Medicina-Universidad de San Martin de Porres. 


\section{INTRODUCCIÓN}

Uno de los principales problemas de salud en nuestro país es la desnutrición, es ampliamente conocido que se presenta por la inadecuada ingesta de nutrientes (deficiente en yodo, hierro y micronutrientes) o por la existencia de una enfermedad recurrente, o la presencia de ambas (1).

Un niño desnutrido es un niño vulnerado es sus potencialidades para poder enfrentar adecuadamente la pobreza, la desnutrición afecta directamente su desarrollo (2).

La literatura sobre las consecuencias de la desnutrición en niños indica, entre otros aspectos, que aumenta el riesgo de morbilidad, mortalidad y deteriora el crecimiento (3). Un niño en etapa escolar reduce sus potencialidades por la desnutrición crónica, se ve afectado su desarrollo físico e intelectual; el impacto de la desnutrición no queda en esta etapa de vida sino que se extenderá hasta la vida adulta. Un niño con desnutrición crónica reducirá su rendimiento académico y en el futuro su capacidad laboral, y desde luego, verá seriamente afectada su capacidad económica (4).

Según el Instituto Nacional de Estadística e Informática la desnutrición infantil rural es $32.3 \%$ y la citadina $10.3 \%$; la región con mayor desnutrición es Huancavelica con $42.4 \%$ frente a Lima Metropolitana que tiene $4.1 \%$.

No soslayemos que la obesidad es un problema mundial de Salud Pública, afecta tanto a los países desarrollados como aquellos en vía de desarrollo. Entre los grupos de mayor riesgo para la obesidad está la población infantil, principalmente la población en edad escolar, en este grupo poblacional es sin duda el hogar el ambiente físico y social de su desarrollo. Con el transcurrir de los años, la interacción del ambiente es más complejo y menos controlado por el hogar; por ello las percepciones y decisiones nutricionales se definen principalmente en el entorno familia, comunidad y escuela (5).

En la Encuesta Nacional de Hogares (ENAHO) del 2008 referentes a datos de prevalencia de niños en edad escolar muestra evidencia razonable para concluir que la obesidad es un problema que se encuentra concentrado en las zonas urbanas principalmente en Lima (11.4\%) más que en las rurales $(2.7 \%)$.

Durante estos últimos años el Estado Peruano en el marco de su modernización y crecimiento económico ha venido asignando importantes recursos a programas de asistencia alimentaria y nutricional a niños en etapa escolar. Los programas públicos han presentado dificultades en su implementación, monitoreo, escasa articulación con actores sociales, filtraciones, duplicidad de acciones y evaluación, redundando ello negativamente en el logro de sus objetivos (6).

El objetivo del estudio fue comparar indicadores antropométricos en dos escuelas primarias de una zona rural y citadina de características similares para determinar la tendencia de desnutrición y sobrepeso en estas zonas.

\section{MATERIAL Y MÉTODOS}

Estudio analítico, prospectivo de corte transversal - comparativo. La población estuvo conformada por niños y niñas procedentes de dos escuelas públicas de una zona citadina (Carabayllo en Lima) y rural (Acobamaba en Huancavelica). Se eligieron dos escuelas públicas con escolares cuyas edades se encontraban entre los 7 a 12 años. La muestra estuvo conformada por 159 niños en Acobamaba y 280 niños en Carabayllo.

Se recolectó información sobre las variables de estudio (edad, sexo, peso, talla e IMC) mediante una ficha pre elaborado. El periodo de recolección de la información fue entre abril y mayo de 2014.

Los datos fueron procesados con el Statistical Package for the Social Sciences v21. Se hizo uso de estadística descriptiva y prueba T para muestras independientes para comparar las medias de IMC de las poblaciones estudiadas (Acobamba y Carabayllo). 


\section{RESULTADOS}

Con referencia a la edad de los escolares, éstas fluctuaban ente 7 a 12 años observando que la media en Carabayllo fue de 10.39 años y en Acobamaba fue 11.01 años.

Asimismo, la proporción de escolares de sexo femenino fueron de $51.07 \%(143 / 280)$ en el distrito de Carabayllo y 50.31\% (80/159) en Acobamaba, no observando valores extremos en la muestra estudiada.

Al evaluar las medidas antropométricas de peso y talla en Carabayllo y Acobamaba se observó que el promedio del peso fue de $31.38 \mathrm{~kg}$ (DS: 1.22 ) y de $30.13 \mathrm{~kg}$ (DS: 5.34 ) respectivamente; mientras que la media de la talla fue $132.58 \mathrm{~cm}$ (DS: 7.1 ) y 132.50 $\mathrm{cm}$ (DS: 8.42 ) respectivamente (Tabla 1 ).

Tabla 1. Características de la población estudiada

\begin{tabular}{|c|c|c|c|}
\hline & CARABAYLLO /1 & ACOBAMBA /2 & $p$ valor \\
\hline Edad media (años) & $10.69+/-1.22$ & $10.59+/-1.60$ & 0.109 \\
\hline Sexo Femenino (\%) & 51.07 & 50.31 & 0.171 \\
\hline Peso medio (kg) & $31.38+/-4.63$ & $30.13+/-5.34$ & 0.125 \\
\hline Talla media (cm) & $132.58+/-7.1$ & $132.50+/-8.42$ & 0.202 \\
\hline \multicolumn{4}{|l|}{ /1 280 niños (as) } \\
\hline /2 159 niños (as) & & & \\
\hline
\end{tabular}

Se realizó la medición de la media del Índice de Masa Corporal (IMC) en los escolares de Carabayllo que fue de 17.85 y de Acobamba con 17.09.

Del análisis estadístico se pudo determinar que los Índice de Masa Corporal (IMC) para los diferentes grupos etarios de 7 a 12 años de edad de los escolares de Carabayllo y Acobamaba no presentan diferencias estadísticamente significativas (Tabla 2).
Tabla 2. IMC medio en la población estudiada según edad

\begin{tabular}{lcccc} 
& CARABAYLLO $/ 1$ & ACOBAMBA $/ 2$ & p valor \\
Niños (as) de 7 años & $15.05+/-1.63$ & $14.72+/-1.42$ & 0.594 \\
Niños (as) de 8 años & $15.64+/-1.64$ & $15.37+/-1.64$ & 0.628 \\
\hline Niños (as) de 9 años & $16.78+/-1.42$ & $16.84+/-1.31$ & 0.858 \\
\hline Niños (as) de 10 años & $18.51+/-1.65$ & $18.49+/-1.84$ & 0.955 \\
\hline Niños (as) de 11 años & $17.93+/-1.17$ & $18.02+/-1.26$ & 0.708 \\
\hline Niños (as) de 12 años & $18.90+/-2.50$ & $17.73+/-2.03$ & 0.058
\end{tabular}

Con los indicadores antropométricos realizados se procedió a la clasificación nutricional observando que los escolares de Acobamba presentaron prevalencias mayores de desnutrición (10.06\%) en comparación a Carabayllo (4.64\%), mientras que la prevalencia de obesidad/sobrepeso en escolares de Carabayllo (16.79\%) fue cercana a la comparada por los escolares en Acobamba (13.84\%), no encontrando diferencias significativamente estadísticas a pesar de ser escuelas ubicadas en zona citadina y rural. (Tabla 3).

Tabla 3. Desnutrición y Obesidad/sobrepeso en la población estudiada

\begin{tabular}{|ccc} 
& DESNUTRICIÓN & OBESIDAD/SOBREPESO \\
\hline CARABAYLLO & $4.64 \%$ & $16.79 \%$ \\
\hline ACOBAMBA & $10.06 \%$ & $13.84 \%$ \\
\hline
\end{tabular}

\section{DISCUSIÓN}

La evaluación antropométrica es la medición de las dimensiones del cuerpo humano en diferentes edades y su comparación con estándares de referencia (7).

A partir de estos se pueden determinar las anormalidades en el crecimiento y desarrollo 
como resultado de conocer el estado nutricional de un individuo o de un grupo que estén en riesgo de alteraciones pudiendo ser utilizados para la identificación de riesgos, intervenciones y evaluaciones de impacto en salud pública.

La nutrición se ha convertido en un tema clave en la discusión del desarrollo económico, no sólo por el impacto que tiene en el crecimiento de un país, sino también porque es comúnmente aceptado que el gobierno tiene la obligación de garantizar un estándar mínimo de vida para todos sus ciudadanos. Los programas de gobierno (Juntos, Qali Warma, etc) deben evaluar periódicamente el impacto sobre la nutrición de la población en pobreza y pobreza extrema dando énfasis en la niños menores de 5 años y la etapa escolar.

Es en este contexto que se realizó las mediciones antropométricas en dos escuelas públicas de diferentes lugares: citadina y rural con características similares para identificar el estado nutricional de escolares de primaria.

Los resultados de prevalencia de desnutrición encontrados en Carabayllo (4.64\%) con referencia a Acobamba (10.06\%) es un reflejo confluyente de estrategias y políticas multisectoriales en mejorar los indicadores de desnutrición crónica infantil y que está asociado posiblemente al desarrollo geográfico de la comunidad influenciada por ser una zona rural o citadina. Sin embargo, esta aproximación debería ser contratada con el análisis de otros variables como la estratificación social y el nivel educativo de la madre (8).

Un hallazgo importante es de que no existen diferencias estadísticas entre los IMC de los escolares de Acobamba y Carabayllo, que a pesar de ser zonas geográficas diferenciadas tengan prevalencias similares, lo cual indicaría que la proporción de mayor prevalencia de obesidad/ sobrepeso encontrado por ENAHO 2008 en zonas urbanas, estén actualmente cambiando y estén equiparando a las prevalencias rurales debido a cambios epidemiológicos en el consumo de alimentos y diversos efectos externos en el consumo de dietas saludables de los escolares.
Los IMC representan una correlación positiva con la adiposidad corporal, a su vez asociado a factores de riesgo para el desarrollo de enfermedades crónicas constituyéndose en una carga de enfermedad potencial en el cambio de los perfiles epidemiológicos de salud en zonas rurales.

Similares resultados se encontraron en la evaluación del estado nutricional de los niños que asistían a un Programa Nacional de guarderías escolares en Chile, detectando una alta prevalencia de la obesidad por la cual los programas de bienestar nutricional no sólo deben evaluar deficiencias nutricionales en base a los parámetros peso-edad y altura para la edad, sino que es necesario a incorporar la medición del indicador peso para la edad que mide obesidad (9).

Debemos empezar a mirar los determinantes de la obesidad/sobrepeso en tres niveles de causalidad: a) inmediata (ingesta alimenticia y estatus de salud), b) subyacente (seguridad alimentaria, cuidado materno-infantil y calidad del entorno de salud) y c) básica (disponibilidad de recursos económicos y entorno político); no sólo (como se hace hoy), concentrarse en los determinantes subyacentes. Por ejemplo a medida que aumenta el número de miembros en una familia, también se debe incrementar la disponibilidad de recursos para adquirir alimentos.

En la mayoría de los casos esto no se logra y, por lo tanto, se pone en riesgo el estado nutricional de todos los miembros de la familia y, especialmente, el de los menores de cinco años (10).

Una visión amplia permitiría entender (entre otras cosas) que la educación de las madres tiene el impacto más fuerte en la desnutrición de los niños, impacto que es seguido por la disponibilidad per cápita de alimentos y el estatus de las mujeres en relación con el de los varones (11).

Es importante destacar que cualquier implementación de política para disminuir la obesidad/ sobrepeso no debe descuidar el desarrollo de modelos de atención comunitarios (12). 
Deben establecerse los lineamientos nacionales para el diseño regional de tales modelos. Sin embargo, ellos deben incluir la participación de actores locales, como organizaciones sociales de base y alcaldes, entre otros, para que sean estos los que validen el modelo y apoyen su gestión (13).

Asimismo, el Estado Peruano debe no sólo adecuar estrategias para la disminución de la desnutrición sino también ir diseñando políticas y propuestas para el control de la obesidad en los escolares en el Perú habiendo indicios que la ruralidad ya no es una barrera de la presencia de este problema de salud pública (14).

\section{REFERENCIAS BIBLIOGRÁFICAS}

1. Vasconcellos M; Anjos L. Estado nutricional e tempo de tela de escolares da Rede Pública de Ensino Fundamental de Niterói, Rio de Janeiro, Brasil. Cad Saude Pública 2013; 29(4): 713-722.

2. Díaz, M; Montoya I. Case study: school meals management in Santiago de Cali and Bogota. Revista de Salud Pública, Bogotá, 2011; 13(5): 737-747.

3. Kain J; Galván M; Taibo M. Evolution of the nutritional status of Chilean children from preschool to school age: Anthropometric results according to the source of the data. Archivo latinoamericano de nutrición 2010; 60(2): 155-159.

4. Mispireta ML. Determinantes del sobrepeso y la obesidad en niños en edad escolar en Perú. Rev Perú Med Exp Salud Pública. 2012;29(3):361-65

5. Kaufer M,Toussaint G. Indicadores antropometricos para evaluar sobrepeso y obesidad en pediatría. Bol Med Hosp Infant Mex. Vol 65: 502-18

6. Ministerio de Salud de Perú, Instituto Nacional de Salud, Centro Nacional de Alimentación y Nutrición. Dirección Ejecutiva de Vigilancia Alimentaria y Nutricional Componente Nutricional en la Encuesta Nacional de Hogares (ENAHOCENAN) 2009 - 2010.

7. López S. Fundamentos de nutrición, $8^{\circ}$ ed. Quito: Dirección Metropolitana de Salud; 2007.
8. Torres M. Social stratification and nutritional anthropometry in children under 15 years old: la escalera Lara State Venezuela. Investigación Clínica 2007; 48(3): 327-340.

9. Gutiérrez Y. Nutritional status of preschool children attending the Chilean national nursery schools council programs (JUNJ): assessment of the agreement among anthropometric indicators of obesity and central obesity. Archivo latinoamericano de nutrición 2009; 59(1): 30-37.

10. Alcaraz G; Bernal C. Nutritional status and living conditions in children in an urban area of Turbo, Antioquia, Colombia. Biomédica 2008; 28(1): 87-98.

11. Caballero E; Blas P. Efecto de una intervención nutricional sobre la prevalencia de anemia en niños de 6 a 9 años de instituciones educativas públicas del Cercado de Lima. Revista de Nutrición 2011; 5(15): 752-763.

12. Zamora A. Rendimiento escolar y estado nutricional. Bogotá: Ministerio de Educación, cultura y deportes Ecuador. Editorial sagitario S.R.L; 2007.

13. Villagrán S. Nutrición y Alimentación en el desarrollo de los niños (as). Ecuador: Universidad Tecnológica Equinoccial; 2009.

14. Restrepo M. La alimentación y nutrición del escolar. [tesis para optar al título de Magister en Salud Colectiva]. Medellín: Universidad de Antioquia; 2008. 116 pp.

\section{Fuentes de financiamiento}

Este artículo ha sido financiado por los autores.

\section{Conflictos de interés}

Los autores declaran no tener ningún conflicto de interés.

\section{Correspondencia:}

Pedro Javier Navarrete Mejía

Dirección: Av. El corregidor 1531, La Molina, Lima -Perú Teléfono: 998998844

Correo electrónico: pnavarretem@usmp.pe 